\title{
Thickness control of thin dielectric layers by the generalized m-line spectroscopy method
}

\author{
E. Auguściuk ${ }^{*}$, B. Bogdanowicz \\ Faculty of Physics, Warsaw University of Technology, Koszykowa 75, 00-662 Warszawa
}

Received May 18, 2010; accepted June 16, 2010; published June 30, 2010

\begin{abstract}
This paper presents an application of the generalized mline spectroscopy method to immediate evaluation of a thin dielectric layer thickness deposited on a waveguide without any numerical calculation. For this purpose, a calibration curve (i.e., dependence of a thin layer thickness on coupling angle of the corresponding mode) shall be determined. It allows determining sample thickness by measuring the coupling angle of the mode. In the present paper it has been shown that multimode waveguides are best for our measurement investigation. The sensor has the greatest sensitivity in the measurement of coupling angles for a higher number of modes.
\end{abstract}

Organic thin layers are used in optoelectronics, both as a conductive and insulating layer in simple passive systems, as well as in more complex systems, such as active MEOMS. It is important to control the parameters and especially the thickness of these layers. The change of thickness of thin monolayers deposited consecutively on the substrate was determined by measuring the contact angle (CA) [1].

The generalized m-line spectroscopy method [2-4] has been proved to be useful in determining with high accuracy of the parameters of thin layers, deposited on the waveguides (with the parameters previously evaluated by m-line spectroscopy method [5-8]).

We can couple a light beam to the waveguide structure, using a prism with a convex angle $\varepsilon$. For the discrete values of angle $\alpha_{\mathrm{m}}$, the light propagates in the waveguide layer in the form of $\mathrm{m}$ modes illustrated on the screen as lines depicted in Figs. 1 and 2. The dark line emerging in the spot (Fig. 1) corresponds to the coupled energy of a light beam.

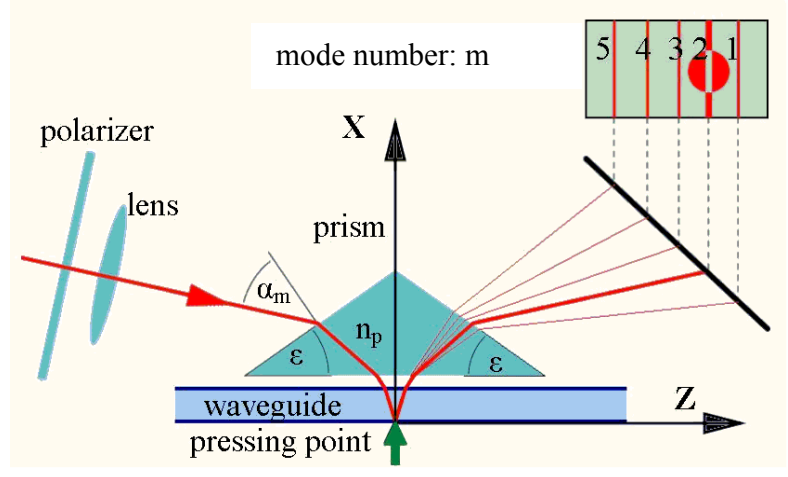

Fig. 1. The coupling by the prism

The propagation constant $\mathrm{N}_{\mathrm{m}}$ can be calculated, based on geometrical optics and its dependence on several parameters as shown in the dispersion equation [2-8]:

$$
N_{m}=N\left(m, n_{f}, W, k, n_{s}, n_{c}\right)
$$

where:

$m$ - mode number,

$n_{f}$ - refractive index of a waveguide,

$\mathrm{W}$ - waveguide thickness,

$\mathrm{k}=2 \pi / \lambda$ - wave vector,

$n_{s}$ - refractive index of a substrate,

$n_{c}$ - refractive index of a cover.

The measurement setup to determine optical parameters as a refractive index and thickness of a waveguide is shown in Fig. 2.

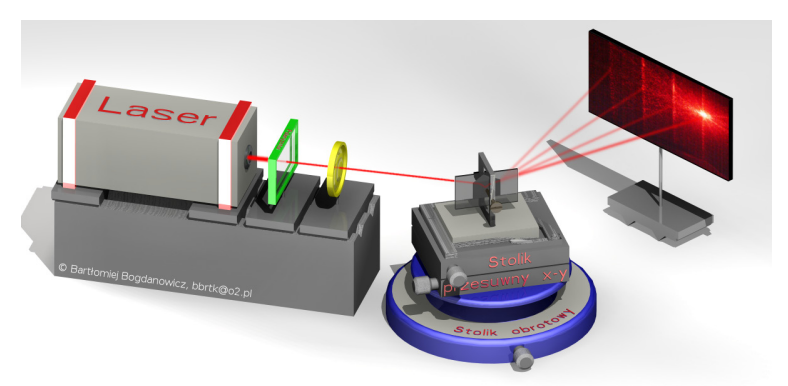

Fig. 2. The measurement setup

*E-mail: eaugust@if.pw.edu.pl 
A light beam from a laser He-Ne with the wavelength of $\lambda=632.8 \mathrm{~nm}$ is propagated by a polarizer and a lens and it is coupled into the waveguide by a prism, in the pressure point (by the screw).

The parameters of a waveguide can be evaluated by the approximation method, within the limits of experimental error [2].

The waveguides were produced by the ion-exchange method $\mathrm{Na}^{+} \Rightarrow \mathrm{Ag}^{+}, K^{+}$[9]. In this case the refractive index $\mathrm{n}_{\mathrm{f}}$ decreases monotonically inwards the waveguide [2] (see Fig. 3). By depositing a thin dielectric layer on the gradient waveguide described above we obtain the structure shown in Fig. 3.
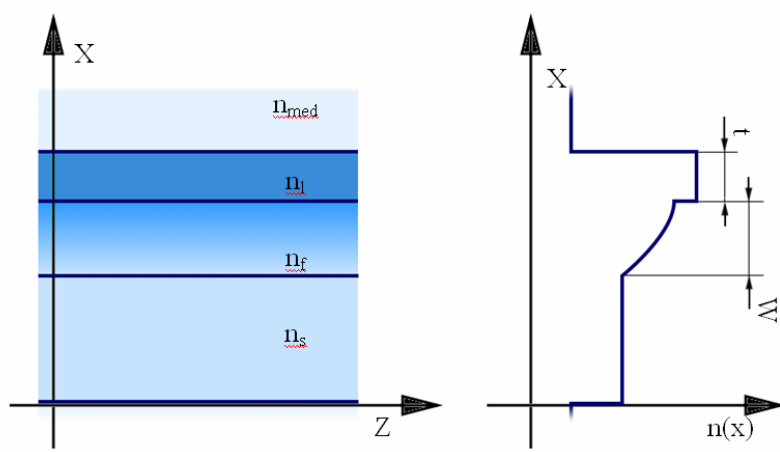

Fig. 3. The waveguide four-layer structure

In several series of measurement, thin polystyrene (PS) layers were deposited on the waveguide by the spincoating method [10]. The layers have a step-index profile of refractive index.

The presence of a thin cover layer on the waveguide changes its propagation characteristics. The generalized m-line spectroscopy method enables the evaluation of the parameters of the sub-guiding layers deposited on a waveguide $[2,11]$.

Table 1 The coupling angles and corresponding layers' thicknesses for AE_ii waveguide with several deposited layers (series)

\begin{tabular}{|c|c|c|c||c|c|}
\hline \multirow{2}{*}{$\begin{array}{c}\text { No sample } \\
\text { mod 1 }\end{array}$} & \multicolumn{4}{|c|}{ Coupling angles } & $\begin{array}{c}\text { Layer } \\
\text { thickness }\end{array}$ \\
\cline { 2 - 6 } & $\left.{ }^{\circ}\right]$ & {$\left[{ }^{\circ}\right]$} & {$\left[^{\prime \prime}\right]$} & {$\left[{ }^{\circ}\right]$} & {$[\mu \mathrm{m}]$} \\
\hline AE_ii_seria_10 & 34 & 42 & 30 & 34,7083 & 1,10 \\
\hline AE_ii_seria_12 & 34 & 54 & 30 & 34,9083 & 1,20 \\
\hline AE_ii_seria_14 & 35 & 10 & 0 & 35,1667 & 1,40 \\
\hline AE_ii_seria_15 & 35 & 33 & 0 & 35,5500 & 1,50 \\
\hline AE_ii_seria_18 & 34 & 35 & 0 & 34,5833 & 1,00 \\
\hline
\end{tabular}

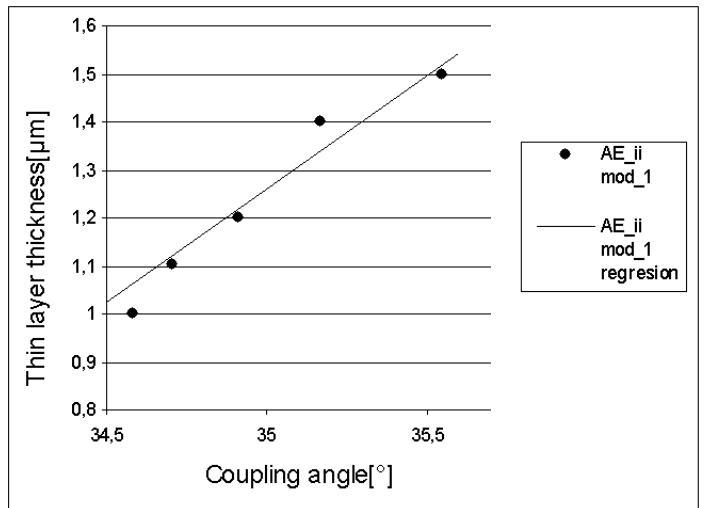

Fig. 4. Calibration curve for the waveguide AE_ii of the first mode.

Figure 4 shows the calibration curve for the waveguide AE ii of the first mode.

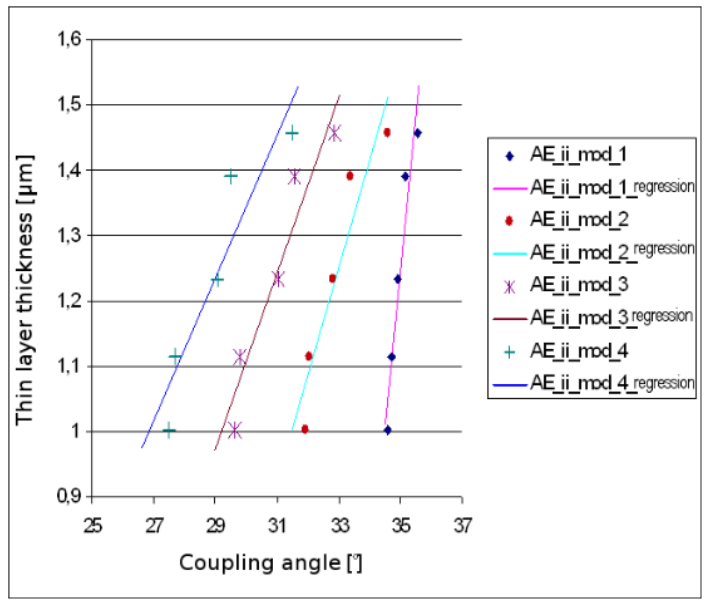

Fig. 5. Calibration curves for four modes of the waveguide AE_ii

Figure 5 shows that the sensitivity of measurement of thickness on the basis of the calibration curve for the 4-th mode is more than 4 times greater than the sensitivity of the measurement for the 1-st mode. So we can conclude that higher sensitivity may be obtained for higher order of modes of the multimode waveguide. Based on Figs. 4-5 one can see a good fit - linear regression according measurement points. The distribution of points suggests the possibility of simple calibration. The distribution of points suggests the possibility of simple calibration. The dependence of a thin layer thickness on the coupling angle of $\mathrm{m}$ mode can be approximated by a linear function and the regression coefficient equals 0.93 .

The AE_ii waveguide characterized during the measurement can be potentially applied as a sensing element of thickness of thin layers (using the generalized m-line spectroscopy method). Using the calibration curve of the sensor (designated by the least squares method) one can determine the unknown refractive index of the 
thin layer. This allows to evaluate the thickness of thin layers, without processing the data obtained by numerical iterative methods, using only the measured coupling angles of the mode. Calibration curves may be more meaningful if the thickness of the thin layers is determined with greater accuracy.

The slopes of a calibration curve for higher order modes are lower, which translates into greater sensitivity. Relative errors that are determined from the slope of calibration curves are of the same order for all the modes. It is estimated that for the construction of the sensor it is best to use multimode waveguides, as well as for the coupling angles of higher order modes.
This work presents a study of the thickness simple sensor system using the generalized m-line spectroscopy method. Avoiding tedious calculations it can determine the thickness of thin layers only by measuring coupling angles and investigating the calibration curve.

\section{References}

[1] E. Auguściuk, M. Roszko, W. Fabianowski, Proc. of SPIE 5064, 245 (2003).

[2] E. Auguściuk, F. Sala, Proc. of SPIE 6585, 65852D (2007).

[3] N. Uchida, Appl. Opt. 15, 179 (1976).

[4] E. Auguściuk, G.Biniecki, Phot. Lett. of Poland 1(3), 124 (2009).

[5] P. K. Tien, R. Ulrich, J. Opt. Soc. Am. 60(10), 1325 (1970).

[6] R. Ulrich, J. Opt. Soc. Am. 60(10), 1337 (1970).

[7] P. K. Tien, Appl. Opt. 10 (11), 2395 (1971).

[8] R. Ulrich, R. Torge, Appl. Opt. 12(12), 2901 (1973).

[9] T. Findakly, Optical Engineering 24( 2), 244 (1985).

[10] http://pl.wikipedia.org/wiki/Spin-coater

[11] E. Auguściuk, Proc. of SPIE 7120, 71200J (2008) 